

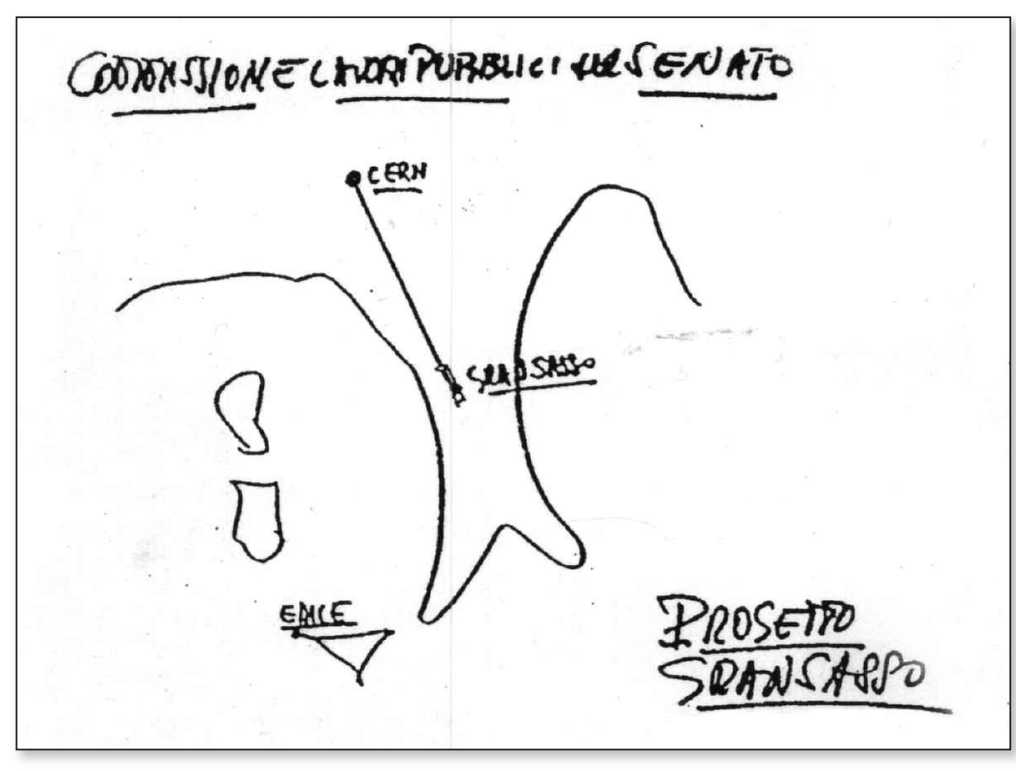

$\Delta$ FIG. 1: Geographical sketch of the neutrino beam from CERN to Gran Sasso by A. Zichichi (1979)

\section{Neutrino oscillations}

Neutrino oscillations happen because neutrinos of definite flavour, $v_{e}, v_{\mu}$ and $v_{\tau}$, are not stationary states, i.e., states of definite mass, but linear combinations of those. We call the latter $v_{1}, v_{2}$ and $v_{3}$ and $m_{1}, m_{2}$ and $m_{3}$ their masses.

Oscillations phenomena are common in physics. Consider for example two identical pendulums, $a$ and $b$, of mass $m$ and length $l$ weakly coupled by a spring of constant $k$. The system has two normal modes, obtained

V FIG. 2: A. Bettini (left) and L. Maiani (right), together with a representative of local Swiss authorities celebrating at the "ground breaking" event for the CNGS project on 12/10/2000. Photo CERN

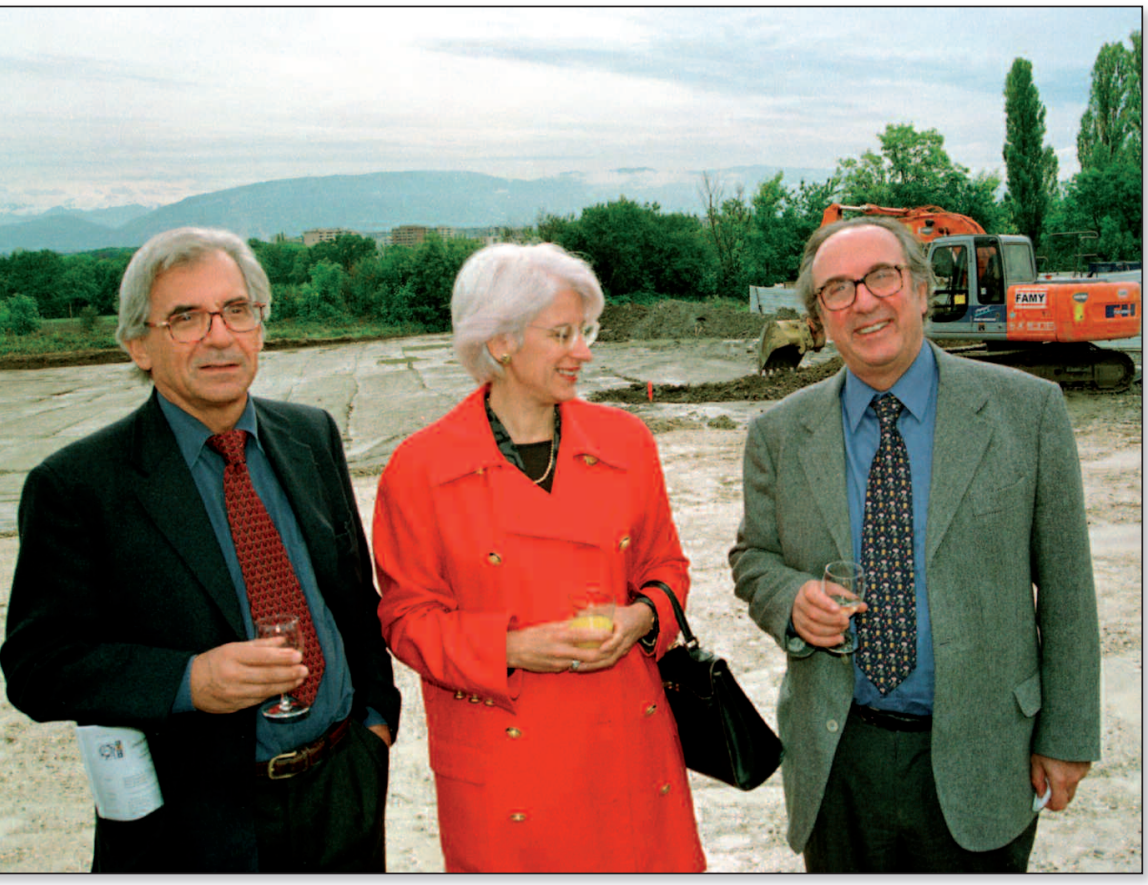

by defining its initial state. In each mode the two pendulums oscillate harmonically and synchronically with (proper) frequencies, say, $\omega_{1}$ and $\omega_{2}$. The first mode is obtained by letting them go from the same initial elongation with zero initial velocities. The squared frequency is $\omega_{1}^{2}=g / l$. In the initial state of the second mode the two elongations are equal and opposite and the velocities are zero. The squared frequency is $\omega_{2}^{2}=g / l+2 \mathrm{k} / \mathrm{m}$. Considering for the moment a world with only two neutrinos, the analogues of the modes are $v_{1}$ and $v_{2}$ and the analogues of the frequencies are the masses $m_{1}$ and $m_{2}$.

The analogue of a neutrino of flavour $a$ is the initial state with pendulum $a$ abandoned with zero velocity at a certain initial elongation and pendulum $b$ in its at rest position. Vice versa for flavour $b$. One observes that the motion does not have a definite frequency. Rather, the amplitude of the vibrations of $a$ gradually decreases down to zero, while that of those of $b$ increases up to a maximum. Then the evolution inverts. Hence, the energy "oscillates" periodically from $a$ to $b$ with a frequency $\omega_{2}-\omega_{1}$. In the quantum system the analogue of energy is the probability to observe the flavour, because both are proportional to the square of the amplitude. To observe the phenomenon one needs to leave a time long enough to allow for its development. In practice this means having the detector far enough from the source. Then there are two alternatives: to look for the disappearance of the initial flavour (looking at $a$ ) or for the appearance of the new one (looking at $b$ ).

However, there are three neutrinos. The analogue is a system of three coupled pendulums. Moreover, the pendulums may not be equal. Clearly the phenomenology becomes much richer.

In 1968 R. Davis [1] published his measurement, started in 1964, of the flux of neutrinos from the Sun with an experiment sensitive to $v_{e}$. The flux was smaller, about $1 / 3$, than the theoretical calculations by J. Bachall [2]. When the preliminary results became known, B. Pontecorvo [3] claimed that the disappearance might be due to neutrinos changing flavour through oscillation, a concept he had introduced in 1957. Further $v_{e}$ disappearance experiments, in different energy ranges, KAMIOKANDE [4] and Super KAMIOKANDE [5] in Japan, GALLEX/GNO [6] at Gran Sasso and SAGE [7] at Baksan, had established by 1998 that the Pontecorvo idea was right. The final proof came from the SNO [8] in Canada in 2002. All neutrino flavours can interact with nuclei producing a neutrino in the final state, instead of a charged lepton. The final neutrino cannot be detected, but the effect on the nucleus can. This was done by SNO, proving that the total neutrino flux interacting in this way corresponded exactly to the missing $v_{e}$ flux. A confirmation came from the KamLAND experiment in 
Japan that observed the oscillation in anti-electron neutrinos from nuclear power plants.

By 1998 the Super-KAMIOKANDE [9] experiment had measured, with high statistics and high accuracy, the dependence of the atmospheric $v_{\mu}$ flux on the flightlength and on energy. The shape of this function proved the oscillation phenomenon and made it possible to determine the corresponding oscillation parameters. Also in this case, confirmation of the oscillation interpretation came from disappearance experiments with artificial neutrinos, $v_{\mu}$ produced at the KEK accelerators in Japan (K2K experiment) and later at the Fermilab in the USA (MINOS experiment).

\section{From the third family to CNGS}

As mentioned above, $v_{\tau}$ and its charged partner $\tau$ are part of the third "family". As a matter of fact, the existence of the third family, and the concept of family itself, was experimentally established in the lepton sector much earlier than in the quark sector. We briefly recall that the neutrino was introduced as a "desperate hypothesis" by Pauli in 1930, when only the electron was known, to explain the apparent non-conservation of energy in beta decay. It was discovered in 1956, when also the muon had been discovered, by F. Raines [10]. It was in 1962 that an experiment at Brookhaven [11] established that neutrinos were two, $v_{e}$ and $v_{\mu}$. Not much later, the idea of the possible existence of a third lepton family, called "heavy lepton" $H_{l}$ and its neutrino $v_{H l}$ was introduced by A. Zichichi. The idea was to search for lepton pairs, which, in the case of $e^{-} \mu^{+}$would be a clear signature of the $H_{l}$. The search started at CERN in 1963, with the PAPLEP experiment, and continued at the $e^{+} e^{-}$collider ADONE [12] at Frascati in 1967. The $H_{l}$ did indeed exist, but was found, and called $\tau$, only in 1975 by M. Perl [13] and collaborators at the SPEAR collider, which, differently from ADONE, had enough energy to produce it.

Thirty years ago, A. Zichichi, then president of INFN (Istituto Nationale di Fisica Nucleare), succeeded in having approved by the Italian Parliament the Gran Sasso project, to build a large, technologically advanced, laboratory under the Gran Sasso massive. The laboratory halls were oriented, in particular, toward CERN, in order to be able in a future to host experiments on a neutrino beam from CERN. The draft presented by Zichichi to the Parliament is shown in Fig. 1.

The vision started to become reality around 1997. Recalling that accelerators produce (almost pure) $v_{\mu}$ beams, the alternative $v_{\mu}$ disappearance vs. $v_{\tau}$ appearance was open. Notice that they require different characteristics both for the beam and the experiments. Vivid discussions started in the community leading to proposals for both. In particular, the OPERA experiment was proposed in that year by A. Ereditato, K. Niwa and P. Strolin [14]. The study of the proposals led to a common decision by the CERN Director General, L. Maiani, the INFN President, E. Iarocci, and the LNGS Director, myself, for the more risky (but much more rewarding if successful) appearance experiments. The project was approved by the INFN and CERN Coun-

\section{Neutrinos do not behave as assumed in the Standard Model (SM): they do change, "oscillate", between one flavour and another}

cils in 1999. It was funded with ad hoc contributions mainly from Italy and from several other countries.

The civil engineering works at CERN and the construction of the beam took place between Autumn 2000 (see Fig. 2) and Summer 2004. The subsequent delicate and complex phases of testing and commissioning were completed by the Spring 2006. In August of the same year the large detectors at LNGS, LVD, OPERA and BOREXINO detected the first events produced by the neutrino beam. In the same period the OPERA and ICARUS experiments were developed.

\section{The first appearance}

As mentioned, the beam produced at CERN is mainly composed of $v_{\mu}$ with no $v_{\tau}$. Consequently, the observation of any $v_{\tau}$ at LNGS must be due to the appearance in the oscillation phenomenon. Experimentally, the two types of neutrinos can be distinguished, when they produce a charged lepton: a $v_{\mu}$ produces a $\mu$ and a $v_{\tau}$ produces a $\tau$. The principle is shown in Fig. 3.

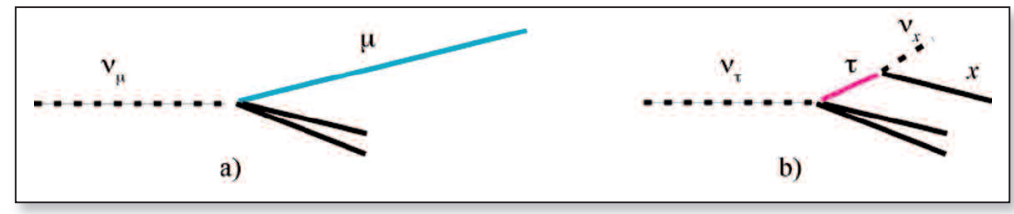

$\Delta$ FIG. 3: Basic topologies of a) $v_{\mu}$ interaction and, b) $v_{\tau}$ interaction

V FIG. 4: The neutrino interaction detection principle of OPERA. Electronic trackers are used to identify the brick containing the interaction, which is then removed and processed.

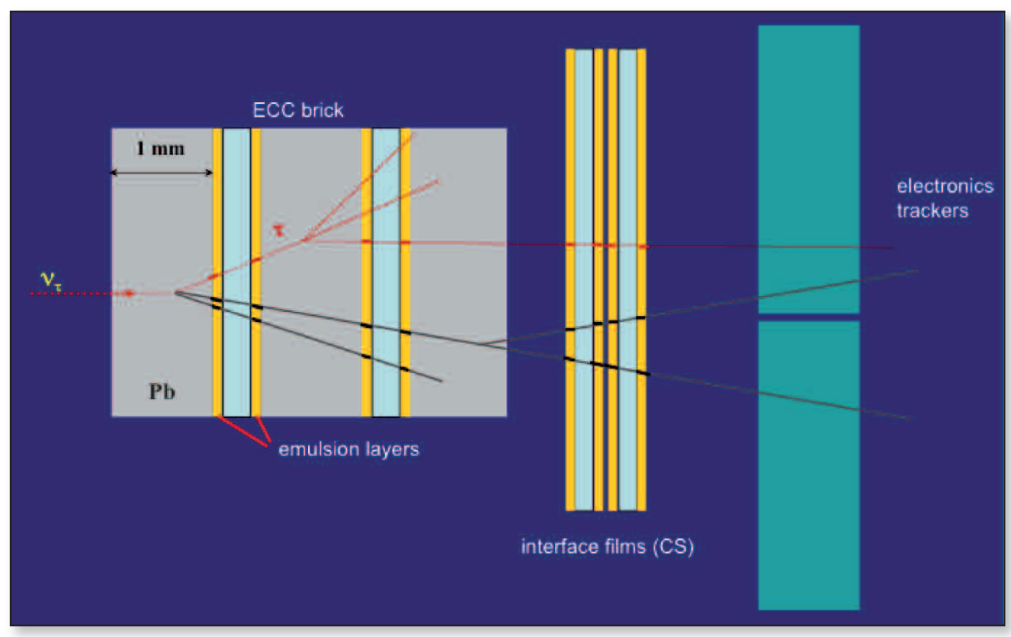


